\title{
Emulation of a Dynamic Broadcasting Network with Adaptive Radiated Power in a Real Scenario
}

\author{
Rodney Martinez Alonso \\ INTEC-WAVES \\ Ghent University \\ Ghent, Belgium \\ rodney.martinezalonso@ugent.be
Glauco Guillen Nieto
$R \& D$ Telecom
LACETEL
Havana, Cuba
glauco@lacetel.cu

\author{
David Plets \\ INTEC-WAVES \\ Ghent University \\ Ghent, Belgium \\ david.plets@ugent.be \\ Luc Martens \\ INTEC-WAVES \\ Ghent University \\ Ghent, Belgium \\ luc1.martens@ugent.be
}

\author{
Yosvany Hervis Santana \\ $R \& D$ Telecom \\ LACETEL \\ Havana, Cuba \\ yosvany@lacetel.cu \\ Wout Joseph \\ INTEC-WAVES \\ Ghent University \\ Ghent, Belgium \\ wout.joseph@ugent.be
}

\author{
Dariel Pereira Ruisanchez \\ $R \& D$ Telecom \\ LACETEL \\ Havana, Cuba \\ dariel@lacetel.cu
}

\begin{abstract}
Broadcasting networks are an efficient means for delivering media content to a high density of users, because their operational cost is almost independent of the size of their audience for a given coverage area. However, when the propagation conditions are better than the worst-case design, the energy efficiency is suboptimal. In this paper, we present the results of a trial to emulate the performance of a dynamic broadcasting network with adaptive radiated power in a real broadcasting scenario. We assess the radiated power of the broadcasting network in a Cuban environment by means of a monitoring device. The power consumption of the dynamic broadcasting network with adaptive radiated power is assessed and compared with traditional broadcasting for different implementation margins. To emulate the performance of the dynamic broadcasting network with adaptive radiated power, we consider a commercial Digital Terrestrial Multimedia Broadcast (DTMB) transmitter in Havana, Cuba. Testbed hardware is designed and developed to measure the fading with a commercial receiver and emulate the signal reception under adaptive power conditions. The dynamic broadcasting network performance is assessed following the general guidelines and techniques for the evaluation of digital terrestrial television broadcasting systems recommended in the ITU-R BT.2035-2 report.
\end{abstract}

Keywords- Dynamic Broadcasting, Adaptive Network, Energy Efficiency, Field Trial

\section{INTRODUCTION}

Broadcasting networks are an efficient means for delivering media content to a high density of users, because their operational cost is almost independent of the size of their audience for a given coverage area [1]. Traditional broadcasting networks are designed considering a worst-case scenario for each service with fixed parameters: i.e., lowest modulation and coding scheme that satisfies a certain bandwidth requirement, and Equivalent Isotopically Radiated Power (EIRP) that guarantee the intended percentage of coverage (e.g, 95\% of locations covered $99 \%$ of the time). When the propagation

Rodney Martinez Alonso is supported by LACETEL and a doctoral grant from the Special Research Fund (BOF) of Ghent University, Belgium. conditions are better than those foreseen in the worst-case design, the energy efficiency of the traditional broadcasting network with fixed EIRP decreases [2].

In the link budget of a broadcasting network design, the shadowing margin $[\mathrm{dB}]$ accounts for the signal variations caused by the topography and obstacles in the propagation environment [3]. The fade margin [dB] accounts for the temporal fading in the transmission channel [4] e.g., caused by the human activity. In a fixed broadcasting network, worst-case margins must be considered to guarantee the Quality of Service (QoS) to the users. However, fading is not constant over time. For instance, in a measurement campaign in Bilbao and Madrid, Spain authors reported an average difference of $3.8 \mathrm{~dB}$ in the fading caused by the vehicular traffic among dense and low traffic locations [5].

In [6], the authors presented a symbiotic collaborative wireless network model capable to adapt to a varying propagation environment or varying network conditions. A spatially adaptive broadcast system capable to adapt the power and antenna patterns through an adaptive algorithm is investigated in [2]. Authors assessed the feasibility of the spatially adaptive broadcasting network by modeling an adaptive system in laboratory conditions. A maximum gain reduction of $4.6 \mathrm{~dB}$ is reported [2]. However, the estimated coverage is significantly reduced as well. For a mean power reduction of approximately $2 \mathrm{~dB}$ the authors observed no coverage reduction [2].

In this paper, we emulate the performance of a dynamic broadcasting network with adaptive radiated power by setting up a trial in a real broadcasting scenario. We assess the radiated power of the broadcasting network, driven by the feedback data from a monitoring device. The power consumption of the dynamic broadcasting network with adaptive radiated power is assessed and compared with traditional broadcasting for different implementation margins. In addition, the trade-off 
between power consumption saving and locations covered is assessed by means of Pareto Optimality.

\section{METHOD}

\section{A. Emulation Setup and Configuration}

Fig. 1 shows a block diagram of a dynamic broadcasting network design.

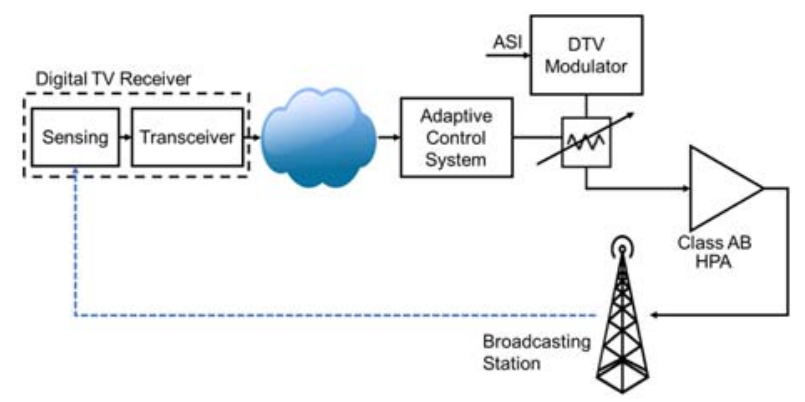

Fig. 1. Block diagram of a dynamic broadcasting network with adaptive radiated power.

In the digital TV receiver, software running in the background in the middleware can measure the signal level variations (e.g., [7]). This information is transmitted to the broadcasting network via a dedicated feedback network. The Adaptive Control System collects the information and accounts for the worst-case reported fading throughout 1 minute (i.e., for $95 \%$ of served locations at a certain instant of time). Hence, the radiated power level at each time stamp (i.e., 1 minute) is correlated with the instant fading by a certain attenuation factor $\left(\beta_{a}\right)$. Equation 1 defines the adaptive radiated power for the dynamic broadcasting.

$$
\operatorname{EIRP}_{a}(t)=P_{o}-L_{R S}+G_{R S}-\beta_{a} \cdot\left[F_{d \max }-F_{d 95}(t)\right]
$$

where $E I R P_{a}(t)[\mathrm{dBm}]$ is the broadcasting network radiated power at a certain instant of time $t, P_{o}[\mathrm{dBm}]$ is the maximum output power of the High-Power Amplifier (HPA), $L_{R S}[\mathrm{~dB}]$ and $G_{R S}[\mathrm{~dB}]$ are the respective losses and gains of the radiation system (i.e., transmission line, couplers, filters, antenna). The difference between the maximum fading (according to the network design) $F_{d \max }[\mathrm{dB}]$ and the instantaneous worst-case fading (i.e., for 95-percent of locations covered) $F_{d 95}(t)[\mathrm{dB}]$, represents the maximum margin of transmit power attenuation that theoretically still satisfies the design constraint (i.e., $95 \%$ of locations covered, $99 \%$ of the time at the cover edge). The attenuation factor $\beta_{a} \in[0: 1]$ is the ratio of attenuation that will be implemented. For $\beta_{a}=1$, the maximum attenuation is applied. Notice that for $\beta_{a}=0$, no attenuation is applied, corresponding to the traditional broadcasting network design.

We assume that the Adaptive Control System can determine which receivers are within the coverage area based on QoS data, i.e., Signal-to-Noise-Ratio (SNR), Bit Error Rate (BER).

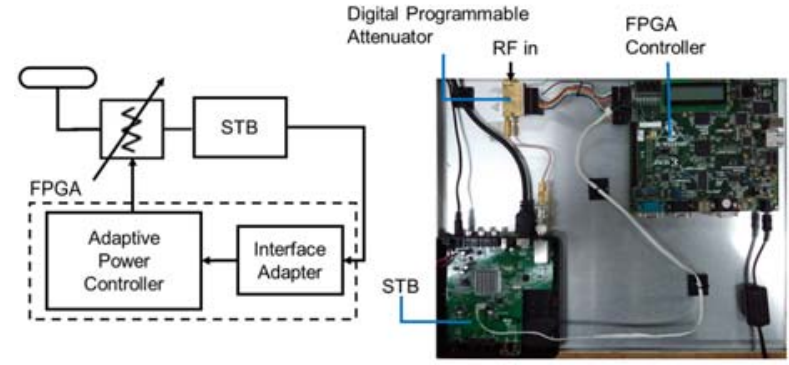

Fig. 2. Emulation testbed hardware.

For maximum power consumption saving, a Class $\mathrm{AB}$ or Doherty High Power Amplifier (HPA) should be considered [8]. This is because Class A amplifiers have an almost constant power consumption independent of the output power. Hence, for a "pure" Class A amplifier, almost no power consumption saving should be expected.

A trial in a broadcasting station requires developing a proper algorithm to protect the HPA from loop related oscillations. This algorithm is highly technology dependent. Hence, the performance of the dynamic network will be emulated at the receiver side. The emulation aims to reproduce, as closely as possible to the real operation, and the environmental and operational conditions of the receivers in the Dynamic Broadcasting Network with adaptive radiated power. For this, testbed hardware based on a commercial receiver is designed and developed to measure the fading, dynamically adapt the signal level (closed-loop control system) and assess the network performance (e.g., coverage, power consumption).

Fig. 2 shows the receiver testbed hardware designed for the trials. Commercial Set-Top Box (STB) middleware is modified to improve signal level measurements. The software algorithm to set-up and adjust the tuner aims to optimize the Signal-to-Noise-Ratio, minimize the distortions, and avoid saturation by adjusting an Automatic Gain Control System (AGC). Although the reception quality is improved, the measurements can be distorted [7]. Hence, the measurement algorithm must be calibrated in order to achieve the highest precision allowed by the receiver hardware.

To compute the fading, the designed software takes 26 power level samples per second as recommended in [5]. The fading is recorded in time windows of 1 minute. The maximum value of fading, not exceeded during more than $1 \%$ of the time at each time stamp, is transmitted to the Adaptive Control System by means of a serial asynchronous interface. The controller will attenuate the signal based on the instant fading and a certain attenuation ratio $\beta_{a}$. Hence, the testbed hardware will emulate the decrease of the signal level, by attenuating the STB input signal proportionally to the current fading conditions. Notice that the signal attenuation is only performed if the QoS related parameters are higher than the minimum thresholds defined for the current modulation and coding scheme. 


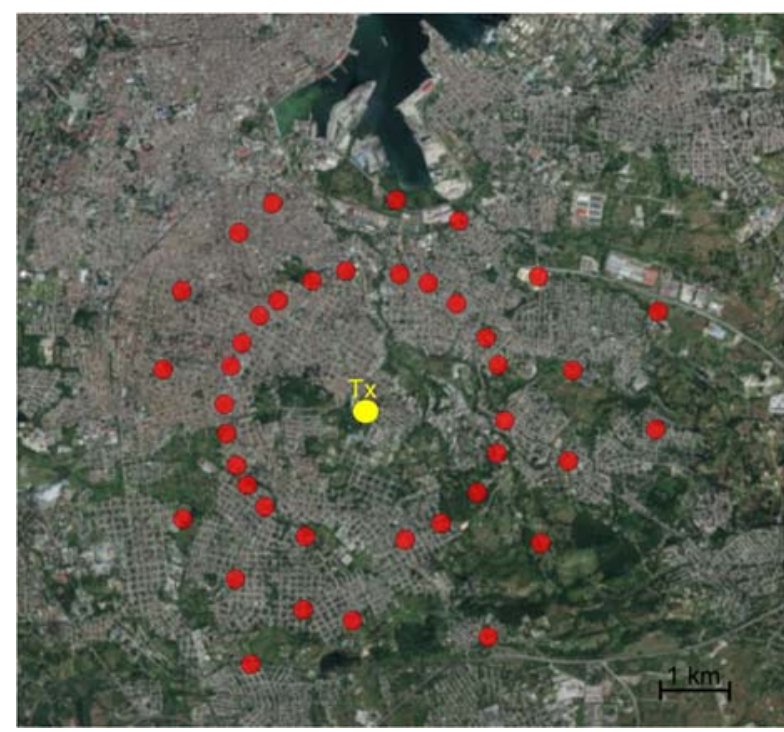

Fig. 3. Broadcast transmitter (TX yellow circle) and 40 measurement locations (red circles).

\section{B. Dynamic Broadcast network coverage assessment}

To emulate the performance of the dynamic broadcasting network with adaptive radiated power, we consider a commercial Digital Terrestrial Multimedia Broadcast (DTMB) [9] broadcasting transmitter in Havana, Cuba, with a coverage area of approximately $47 \mathrm{~km}^{2}$. The peak power of the transmitter HPA is approximately $600 \mathrm{~W}$ with an average power of approximately $100 \mathrm{~W}$ (for DTMB operation). The absolute gain of the radiation system, accounting for antenna gain, feeder losses and coupling efficiency is $8.5 \mathrm{~dB}$. Hence the maximum EIRP is approximately $58.5 \mathrm{dBm}$.

Fig. 3 shows a map with the transmitter location and measurement locations. The broadcasting network with adaptive radiated power performance is assessed following the General Guidelines and Techniques for the Evaluation of Digital Terrestrial Television Broadcasting Systems recommended in the ITU-R BT.2035-2 report [10]. Forty locations around the transmitter were chosen to perform the measurements. The selection of the locations follows a radial distribution (Fig. 3). Inner radials are separated $20^{\circ}$. Due to the relatively low power of the transmitter, the inner circumference is located at $2 \mathrm{~km}$ from the transmitter instead of the $3 \mathrm{~km}$ suggested in ITU-R BT.2035-2 report [10].

At each location, the testbed hardware measured the current fading. The Adaptive Power Controller applies a certain attenuation given the attenuation ratio $\left(\beta_{a}\right)$ settled. We consider 5 values of $\beta_{a}\{0,0.25,0.5,0.75,1\}$. We monitored that the SNR is above the minimum required value $(\sim 15 \mathrm{~dB})$ for the current DTMB transmission mode [11].

The system margin is obtained by introducing an additional controlled attenuation to the input signal until reaching the Threshold of Visibility (TOV) [10]. The TOV is considered to be reached when a trained observer is able to detect some kind of artefact on the image after 1 minute of observation [10]. However, this additional attenuation could be perceived and measured as a fading variation by the receiver. Hence, the fading measurement following the introduction of this additional attenuation will be discarded. A certain location is considered to be covered by the network if the system margin is higher than the maximal fading measured for each value of $\beta_{a}$. Notice that if the system margin is lower than the fading measured at the evaluated location, the user will probably perceive distortions in the transmitted video.

We assume the worst-case scenario, i.e., the current location defines the worst reported fading at the current instant of time. This means, $95 \%$ of the remaining locations (within the coverage area) reported a lower fading at the current timestamp. Hence, we assess the critical condition for each measurement point.

The worst location in terms of maximum fading will define the system performance in a real scenario. Hence, the fading is measured during 24 hours at the worst location evaluated (i.e., higher 99-percentile fading at any instant of time).

The system $E I R P_{a}$ for each $\beta_{a}$ is calculated by means of Equation 1, based on the 24 hours measured fading in the worst location identified. By means of Pareto Optimality, the tradeoff between the average $E I R P_{a}$ and the percentage of locations covered given each value of $\beta_{a}$ is assessed.

\section{Dynamic Broadcast HPA power consumption}

The power consumption of the HPA at each time stamp is calculated by means of Equation 2:

$$
P C_{H P A}(t)=\frac{P_{L}(t)}{\eta_{H P A}\left[P_{L}(t)\right]}
$$

where $P C_{H P A}(t)[\mathrm{W}]$ is the power consumption at a certain instant of time $t, P_{L}(t)$ is the HPA output power, and $\eta_{H P A}$ is the HPA efficiency. We will assess the Power Consumption for two HPA technologies, based on Class AB amplifier [12] and Doherty technology amplifier [13]. Notice that the efficiency of the amplifier is not constant and depends on the power load $P_{L}(t)[\mathrm{W}]$.

Fig. 4 shows the power consumption $P C_{H P A}$ of a Class $\mathrm{AB}$ amplifier and a Doherty amplifier as a function of the amplifier's output power. The theoretical efficiency of a Class AB broadcasting HPA is $78 \%$ for peak power [12]. However, for OFDM broadcasting applications, the average power is typically $1 / 6$ of the peak power (considering a Crest Factor of $8 \mathrm{~dB}$ ) where the amplifier has an efficiency up to $30 \%$ [12]. Under typical broadcast operation, a state-of-the-art Class AB HPA achieves an overall efficiency of $28 \%$ [8]. Notice that below $20 \mathrm{~W}$, the power consumption increases. This is because the efficiency drops exponentially. Hence the minimum allowable output power of the amplifier should be constrained. In addition, the minimum exciting input power of the amplifier must be constrained for the dynamic broadcast network design. 


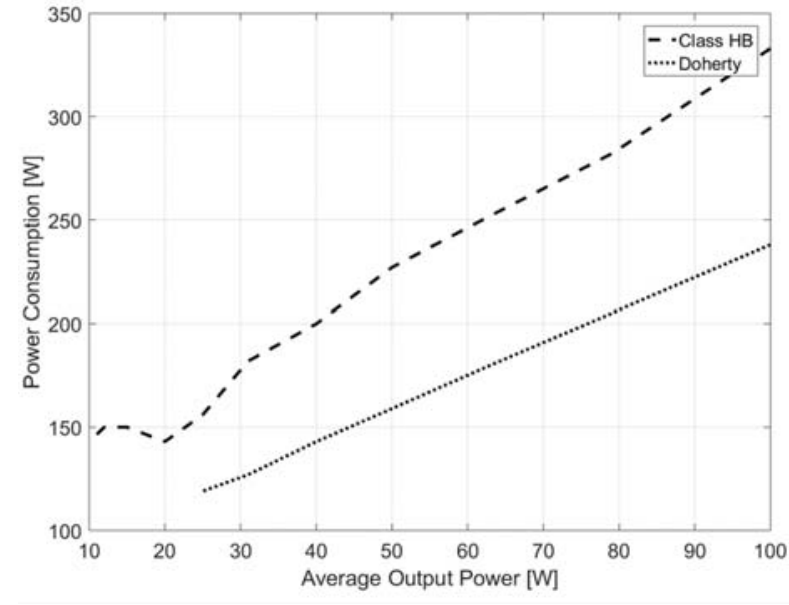

Fig. 4. Power Consumption as a function of Average Output Power (i.e., OFDM signal) of a Class AB HPA and a Doherty HPA [12], [13].

In [13], the authors presented a wideband broadcast Doherty HPA with a peak power of $700 \mathrm{~W}$. This means the average power is around $115 \mathrm{~W}$, which fits in our application. State-ofthe-art Doherty HPAs have a typical operational efficiency up to $42 \%$ [8].

\section{RESULTS}

\section{A. Fading and Coverage measurements}

Fig. 5 shows the recorded fading during 24 hours for the worst location. The 99-percentile fading $\left(F_{d \max }\right)$ in the worst location is $8 \mathrm{~dB}$. However, the 99-percentile fading is lower than $3 \mathrm{~dB}$ on $50 \%$ of the time stamps. Notice that the worst fading during the day occurs from 09:00 to 11:00 and from 16:00 to 18:00. During the worst interval in the morning, the average fading is $\sim 6 \mathrm{~dB}$ ( $3 \mathrm{~dB}$ higher than the 24 hours average) and $\sim 7 \mathrm{~dB}$ in the worst interval in the afternoon $(4 \mathrm{~dB}$ higher than the 24 hours average). There is no a correlation between the fading results and meteorological variables. The 24 hours temperature variation was $8^{\circ} \mathrm{C}$. Hence, the Thermal Noise variation is just $0.1 \mathrm{~dB}$. Also, there was no rain $(0 \mathrm{~mm})$ and the maximum wind speed was $11 \mathrm{~km} / \mathrm{h}$. The wind speed variation over time is not correlated with the fading results. The worst fading was measured in locations near highways with dense vehicular traffic, in this case near a highway connecting the outskirt and the downtown. The worst time lapses match with the highest traffic density during the day.

By means of Equation 1 is possible to calculate the adaptive $E I R P_{a}$ in the transmitter for each value of $\beta_{a}$. Fig. 6 shows the Pareto Optimality graph of the measurement results of the reduction on locations covered as a function of the average adapted EIRPa. The highest average $E I R P_{a}$ reduction is achieved for $\beta_{a}=1$. Nevertheless, because of the STB measurement error, the delay between measurements, and instant fast fading variations, the percentage of locations covered is reduced approximately by $22 \%$ compared with the traditional broadcasting network. For the most conservative value of $\beta_{a}=0.25$, there is no relevant reduction of locations covered and the average $E I R P_{a}$ is reduced by $0.8 \mathrm{~dB}$.

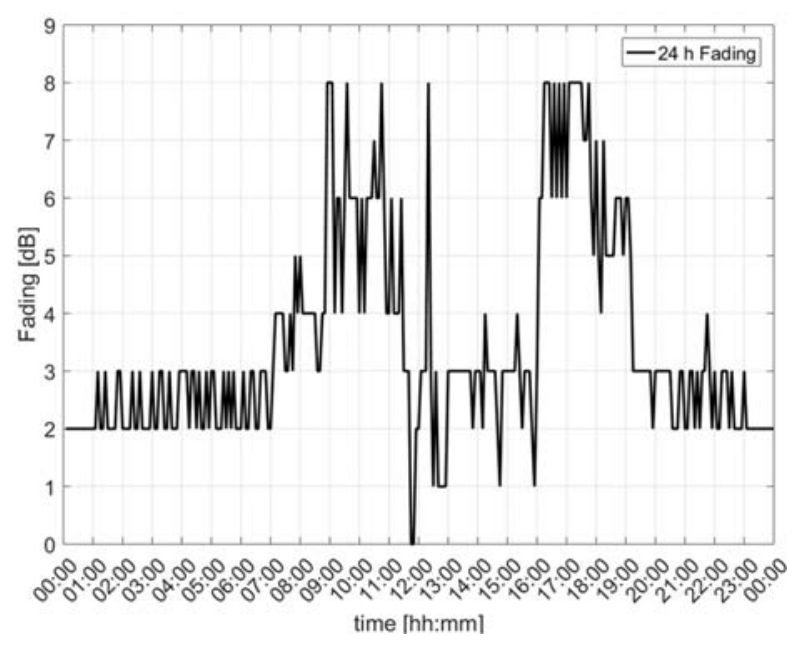

Fig. 5. Worst fading throughout 24 hours.

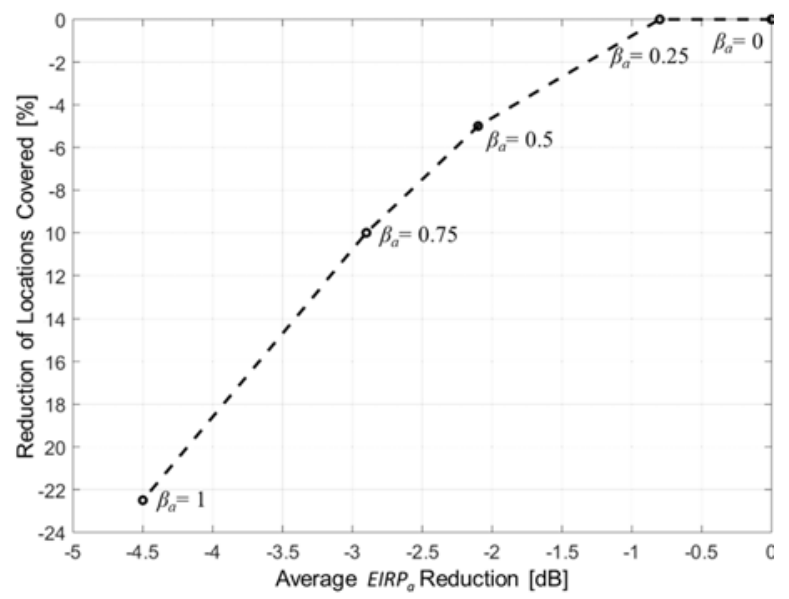

Fig. 6. Pareto Optimality for Reduction of Locations Covered as a function of Average $\operatorname{EIRP}_{a}$ Reduction.

During the measurement trial, the main impact on the reduction of locations covered was caused by fast fading variations caused mainly by vehicular traffic patterns. Most of the locations without coverage correspond to areas with high vehicular traffic (i.e., highways or near to highways). The vehicular traffic patterns cause a variation of the doppler effect and as a consequence, the signal fading variations. The fading variation with vehicular traffic in the UHF band has been also reported in a measurement campaign in [5].

In comparison with the experimental results obtained by authors in [2], the reduction of locations covered is approximately $5 \%$ higher for the same mean power reduction, due to the system lacks to respond to fast fading transitions in a real broadcasting scenario.

Notice that by increasing the total radiated power, the coverage is extended but the current results are not significantly improved. In addition, a longer sampling window (i.e., the signal is sampled during a longer period), could improve the coverage but the $E I R P_{a}$ reduction is lower. Hence, the Pareto Optimality curve is not improved. 


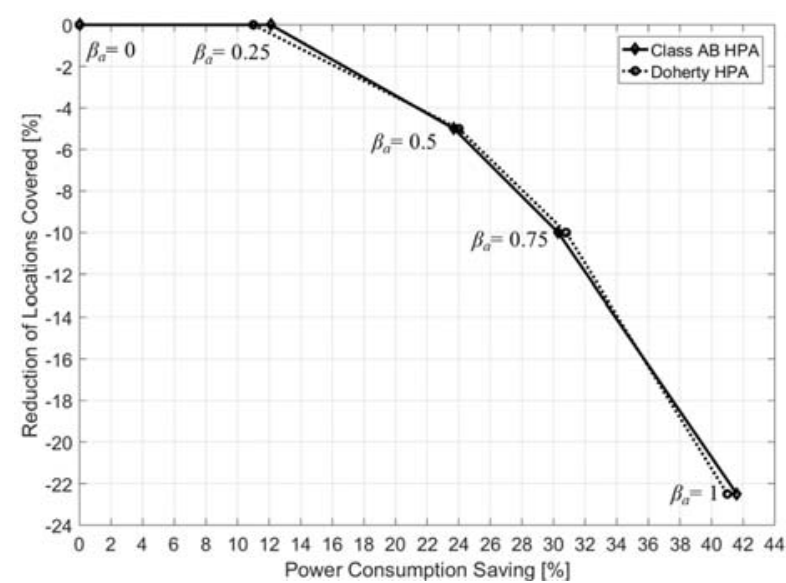

Fig. 7. Pareto Optimality for Reduction of Locations Covered as a function of Average Power Consumption Saving.

\section{B. HPA power consumption assessment}

Fig. 7 shows the Pareto Optimality for Reduction of Locations Covered as a function of the percentage of the HPA Power Consumption saving, compared with the traditional broadcasting network. The power consumption saving is calculated by means of Equation 2, from the difference between a Class AB HPA with fixed EIRP and the same Class AB HPA with adaptive $\operatorname{EIRP}_{a}$. The same calculation is performed for the Doherty technology HPA. We account here only for the Power Consumption of the HPA and no other power consuming components of the Broadcast Transmitter.

The power consumption saving of the HPA between the dynamic and the traditional broadcasting network is higher than $40 \%$ for $\beta_{a}=1$. However, the percentage of locations covered is reduced by $22 \%$. The power consumption of the Class $\mathrm{AB}$ HPA is reduced by approximately $12 \%$ for $\beta_{a}=0.25$ without a significant coverage reduction. For the same value of $\beta_{a}$, the power consumption of the Doherty amplifier with adaptive $E I R P_{a}$ is reduced by $11 \%$. There is no relevant difference in the power consumption saved with the adaptive radiated power solution for the Class AB and Doherty HPA. This is because the power consumption functions of both HPAs have similar slopes in the range of the average output power in this application (see Fig. 4).

The HPA represents approximately $28 \%$ of the broadcasting station power consumption. The main power consumption component of the broadcasting station is the air-cooling $(\sim 70 \%$ of total power consumption). If no dynamic adaptation is implemented in the air-cooling systems the power consumption saving of the whole dynamic broadcasting station is $3 \%$ to $12 \%$.

\section{CONCLUSIONS}

In this paper, we investigated the feasibility of a dynamic broadcasting network with adaptive radiated power by an emulation trial in a real broadcasting scenario.

The EIRP $P_{a}$ of the dynamic broadcasting network can be reduced by $0.8 \mathrm{~dB}$ without a relevant reduction of locations covered. This represents a reduction in the HPA power consumption of approximately $12 \%$. Notice that the main power consuming component of a broadcasting station is the aircooling system $(\sim 70 \%)$. If no air-cooling dynamic adaptation is implemented the power consumption saving of the HPA represents a power consumption saving of approximately $3 \%$. For higher power consumption savings, the locations covered is reduced by $5 \%$ to $22 \%$ compared to a traditional broadcasting network. An improvement of the Adaptive Control System, capable to efficiently respond to fast fading could improve current results.

\section{ACKNOWLEDGMENT}

We would like to thank Jose Alvarez Marin and Laura Quesada del Busto for their kind support in order to carry out the measurement campaign.

\section{REFERENCES}

[1] J. Qi, P. Neumann and U. Reimers, "Dynamic Broadcast," in 14th ITG Conference on Electronic Media Technology, Dortmund, Germany, 2011.

[2] P. Bagot, M. Beach, A. Nix, J. McGeehan and J. Boyer, "Spatially Adaptive TV Broadcast System: Hardware in the Loop Operational Analysis," IEEE Transactions on Broadcasting, vol. 64, no. 1, pp. 41-51, 2018.

[3] W. Joseph, D. Plets, L. Verloock, E. Tanghe, L. Martens, E. Deventer and H. Gauderis, "Procedure to Optimize Coverage and Throughput for a DVB-H System Based on Field Trials," IEEE Transactions on Broadcasting, vol. 54, no. 3, pp. 347-355, 2008.

[4] D. Plets, W. Joseph, E. Tanghe and L. Martens, "Evaluation of temporal fading characteristics of a DVB-H signal for different reception categories," in 2009 IEEE Antennas and Propagation Society International Symposium, Charleston, SC, 2009.

[5] P. Angueira, M. Velez, D. d. 1. Vega, A. Arrinda and J. L. Ordiales, "Fading Caused by Moving Vehicles near the Receiver on DTV (COFDM) 8 MHz Signals," IEEE Communications Letters, vol. 6, no. 6, pp. 250-252, 2002.

[6] D. Plets, W. Joseph, E. D. Poorter, L. Martens and I. Moerman, "Concept and framework of a self-regulating symbiotic network," Wireless Com Network, no. 340, pp. 1-9, 2012.

[7] Y. Hervis Santana, D. Morejon Betancourt, A. I. Mena Valdes, Y. Hernandez Sanchez, G. Guillen Nieto and R. Martinez Alonso, "DTMB monitoring tool based on a commercial Set-Top Box," IEEE International Symposium on Broadband Multimedia Systems and Broadcasting, pp. 1-6, 2017.

[8] Rohde \& Schwarz, "R\&S THU9/R\&S THV9 Liquid-cooled transmitter families," Munich, Germany, 2016.

[9] Standardization Administration of China, "GB 20600-2006 Standard: Framing Structure, Channel Coding and Modulation for Digital Television Terrestrial Broadcasting System," Beijing, China, 2006.

[10] ITU-R, "BT.2035-2 Report: Guidelines and techniques for the evaluation of digital terrestrial," Geneva, 2008.

[11] A. Martinez Alonso, R. Martinez Alonso and G. Guillen Nieto, "Parámetros de calidad de recepción del estándar DTMB en $6 \mathrm{MHz}$," in IEEE Biennial Congress of Argentina, Bariloche, Argentina, 2014.

[12] Rohde \& Schwarz, "The next level of efficiency for broadcasting amplifiers," Munich, Germany, 2013.

[13] J. H. Qureshi, W. Sneijers, R. Keenan, L. C. N. d. Vreede and F. v. Rijs, "A 700-W Peak Ultra-Wideband Broadcast Doherty Amplifier," in IEEE MTT-S International Microwave Symposium (IMS), Tampa, USA, 2014. 